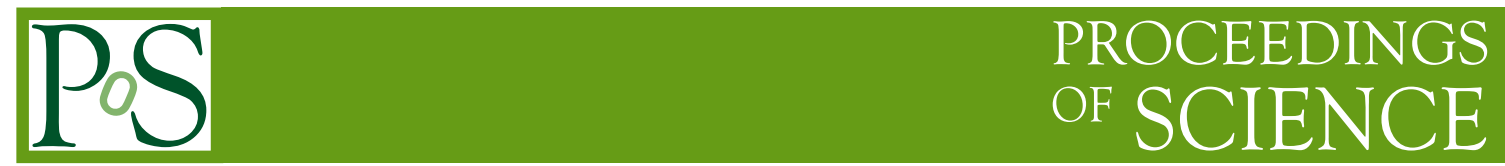

\title{
Status and recent highlights from CMS
}

\section{Petra Van Mulders *}

Vrije Universiteit Brussel (BE)

E-mail: pvmulder@cern.ch

The LHC Run-2 at a center of mass energy of $13 \mathrm{TeV}$ started in 2015. This proceeding highlights some of the physics results based on the collision data collected by the CMS experiment in 2015. In addition, the status and readiness of the experiment for the collisions in 2016 are discussed with concrete examples on the object reconstruction performance.

Fourth Annual Large Hadron Collider Physics

13-18 June 2016

Lund, Sweden

${ }^{*}$ for the CMS collaboration 


\section{Introduction}

The CMS Collaboration consists of over 4400 members from over 200 institutes worldwide. Activities focus mostly on the analysis of the LHC Run 2 collision data, but also on the preparation of the Phase I and Phase II upgrades of the CMS experiment, with the current director described in detail in Ref. [1]. In addition, a number of LHC Run 1 physics analyses is ongoing, in particular for standard model precision measurements. This document focuses on the first Run 2 physics results using the 2015 collision data as well as the readiness for the analysis of the collision data in 2016. There were over 90 physics results released based on the 2015 collision data and around 10 of those were submitted for publication. From the 90 new physics results, only a subset will be discussed.

\section{Physics highlights based on the 2015 collision data}

\subsection{Standard model physics and precision measurements}

The proton collision data at a center of mass energy of $13 \mathrm{TeV}$ opens not only a unique opportunity to discover new physics phenomena but also a to scrutinize the standard model through precision measurements. Figure 1 presents an overview of the production cross section measurements for a wide variety of processes at three center of mass energies. The production cross section measurements are typically performed in different decay channels which are then combined. As

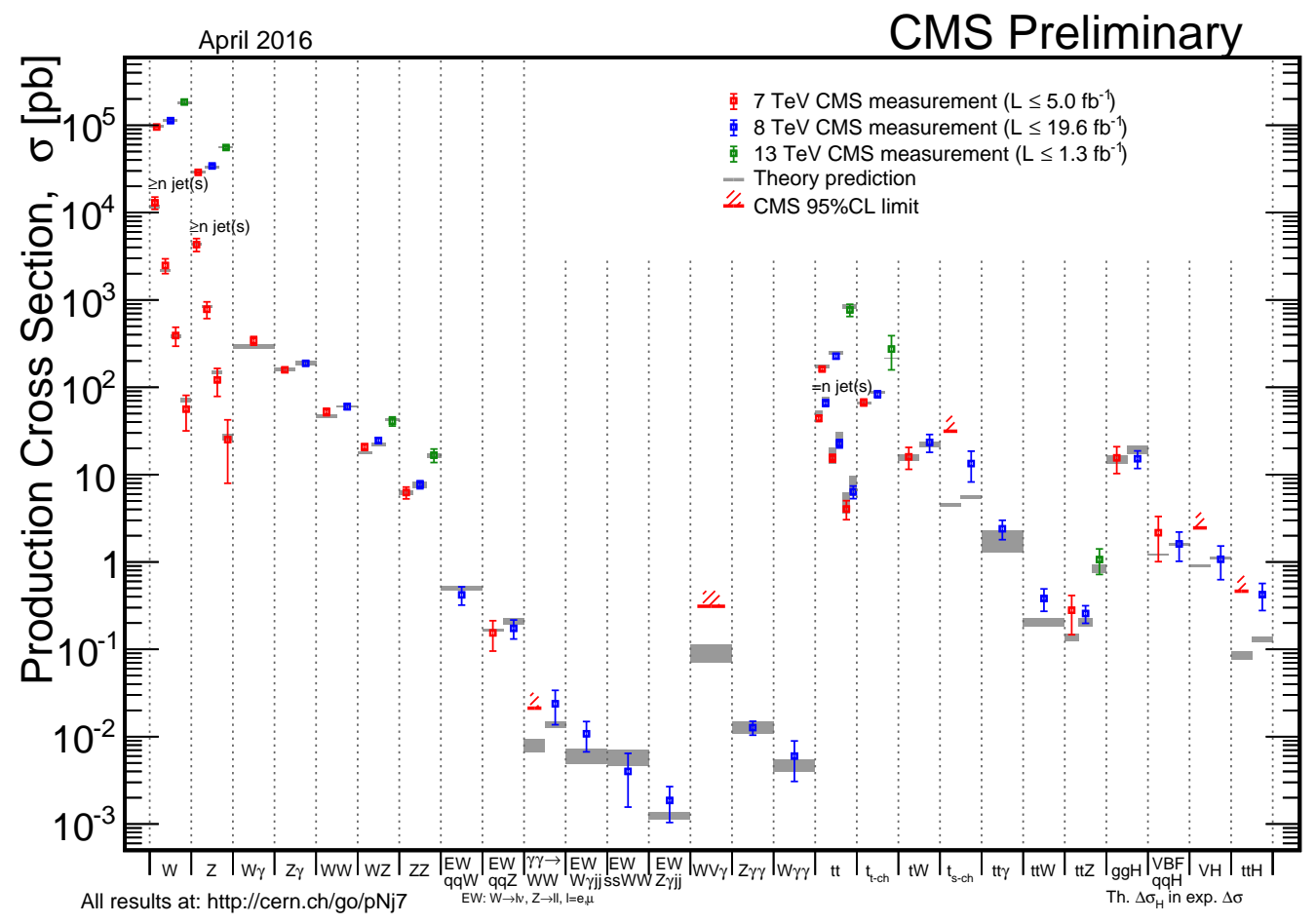

Figure 1: The various production cross section measurements performed by the CMS Collaboration at three different center of mass energies are compared to their prediction.

can be seen, the measurements are in perfect agreement with the predicted cross sections. For some of the processes the sensitivity is not yet sufficient for observation, which is the case for instance 
for the production of a Higgs boson in association with a top quark pair. In such cases the 95\% confidence level upper limit on the production cross section is shown. Whenever the size of the dataset allows it, differential cross section measurements are performed. An example of such a measurement is shown in the left panel of Figure 2, where the differential cross section of inclusive jet production is shown in a bin with jet rapidity below 0.5 as a function of the jet transverse momentum. The measured inclusive jet cross section is best described by the Monte Carlo event generators with next-to-leading order predictions matched to parton showering, hadronisation, and multiparton interactions [2]. This indicates that jet physics is as well understood at $13 \mathrm{TeV}$ as at the smaller LHC Run-1 center of mass energies. The right panel of Figure 2 shows the $\mathrm{W}$ and $\mathrm{Z}$ boson
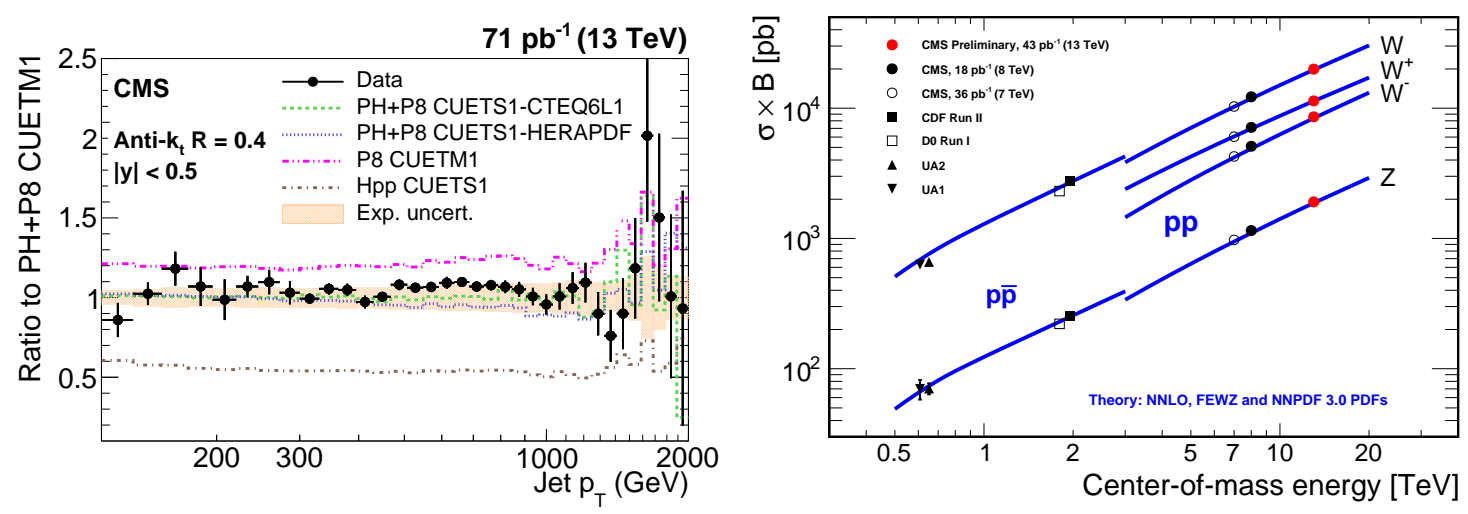

Figure 2: Left: Production cross section for $\mathrm{W}$ and $\mathrm{Z}$ boson as a function of the center of mass energy [2]. Right: Measurement of inclusive jet differential production cross section [3].

production cross section measured at different center of mass energies, including the preliminary result of the CMS collaboration at $13 \mathrm{TeV}$. The measured $\mathrm{W}$ and $\mathrm{Z}$ boson production cross section values agree with the next-to-next-to-leading-order QCD predictions [3]. A similar conclusion can be made for the top quark pair production cross section as shown in Figure 3 [4].

A particularly interesting area is the Higgs boson sector in the standard model. The left panel in Figure 4 shows the fiducial cross section for a Higgs boson decaying to four charged leptons as a function of the center of mass energy [5]. While the statistical precision achievable with the 2015 dataset is limited, the sensitivity of the Run-2 analyses will quickly surpass that of Run-1. Precise measurements of the properties of the Higgs particle will become possible due to the increase in the Higgs boson production cross section at $13 \mathrm{TeV}$ and the large amount of data to be collected. One of the most important goals of the LHC Run-2 is the observation and measurement of the Higgs boson production in association with a top quark pair $(\mathrm{ttH})$, since this process will give important insights on the scale at which new physics is expected. The right panel in the same figure shows the sensitivity for this process obtained with the proton collision data collected in 2015 [6]. Many more recent standard model precision measurements were obtained and are presented in other contributions to these conference proceedings.

\subsection{Searches for beyond the standard model particles}

Apart from the standard model measurements, we also have many results for those physics phenomena that are in a search strategy mode rather than a measurement mode. Only a few of the 


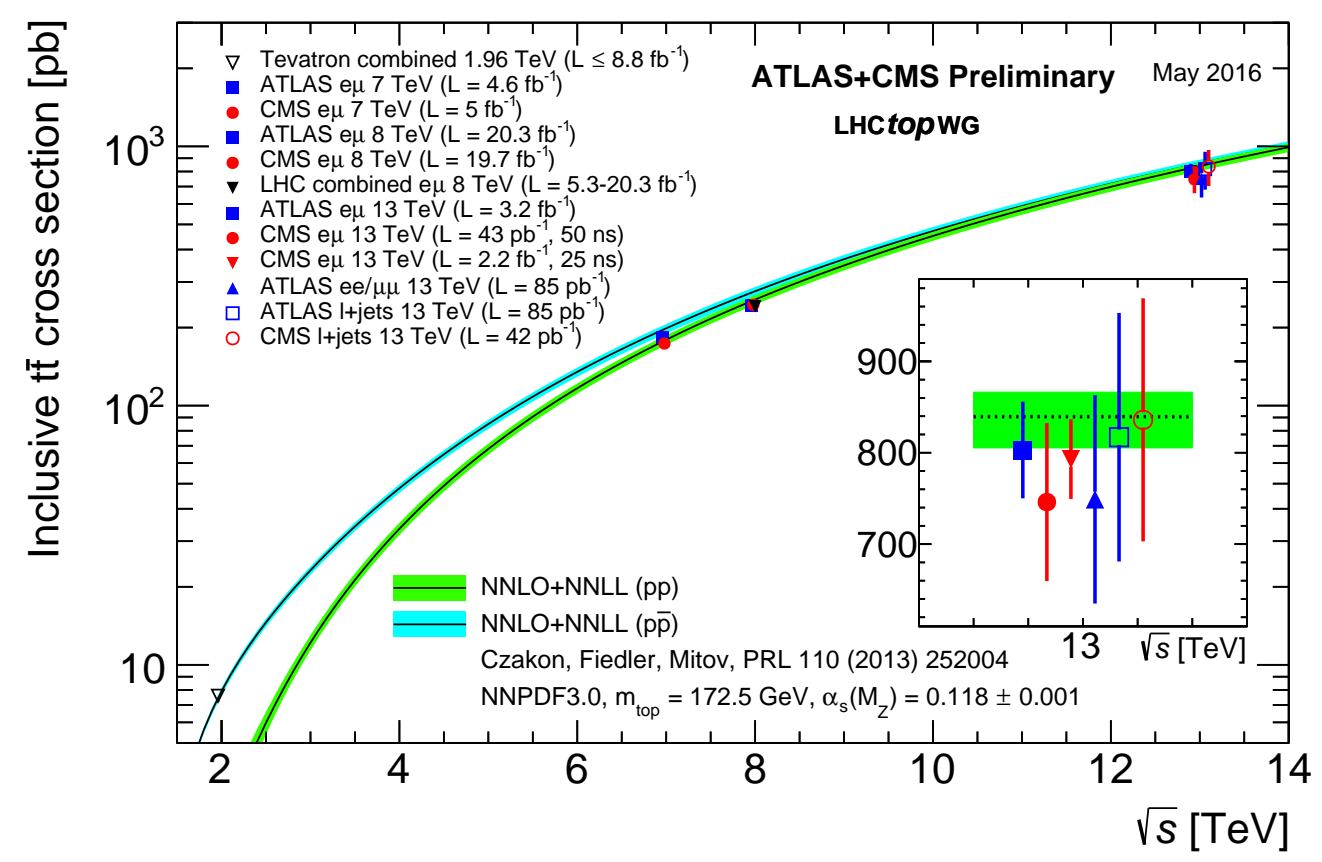

Figure 3: Top quark pair production cross section as a function of the center of mass energy. The inset shows the results obtained at a center of mass energy of $13 \mathrm{TeV}$ [4].
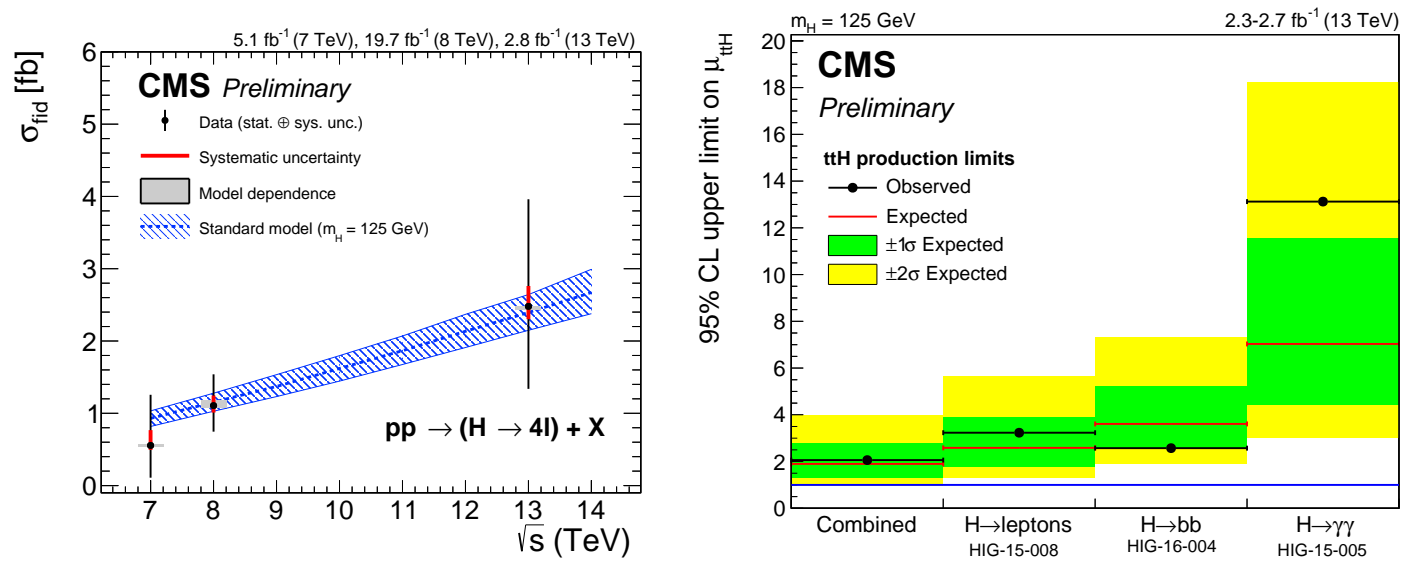

Figure 4: Left: Measurement of the fiducial production cross section for a Higgs boson decaying to four charged leptons as a function of the center of mass energy [5]. Right: 95\% confidence level upper limit on the rate of $\mathrm{ttH}$ production relative to the standard model expectation [6]. 
rich variety of searches recently performed are highlighted here.

Some of the popular extensions of the standard model involve heavy partners of the third generation quarks or heavy gauge bosons decaying to the third generation quarks. An example is the search for a top quark partner with charge $5 / 3$ performed using events with same-sign dileptons and a single lepton [7]. No significant excess is observed in the data above the expected standard model background. The $95 \%$ confidence level upper limit on the production of a right-handed top quark partner with charge 5/3 is presented in the left panel of Figure 5. This search is already surpassing the sensitivity of the corresponding Run-1 search. A second example is the search for the production of heavy resonances decaying to top quark - antiquark pairs in the all-hadronic channel, focusing on high-mass resonances decaying to top quarks with high Lorentz boosts. These highly-boosted top quarks are reconstructed as single jets with substructure corresponding to the $t \rightarrow b W \rightarrow b q q$ decay. No excess above the expectation from the standard model is observed, and 95\% confidence level upper limits are set on the production cross sections of $Z$ bosons and RS gluons, for signal models with varying widths. For wider $Z$ signal models, the exclusion limits are more stringent than the Run-1 limits, excluding $Z$ bosons with masses up to 3.3 (3.8) TeV, for $Z$ widths of $10 \%$ (30\%) of their masses [8].
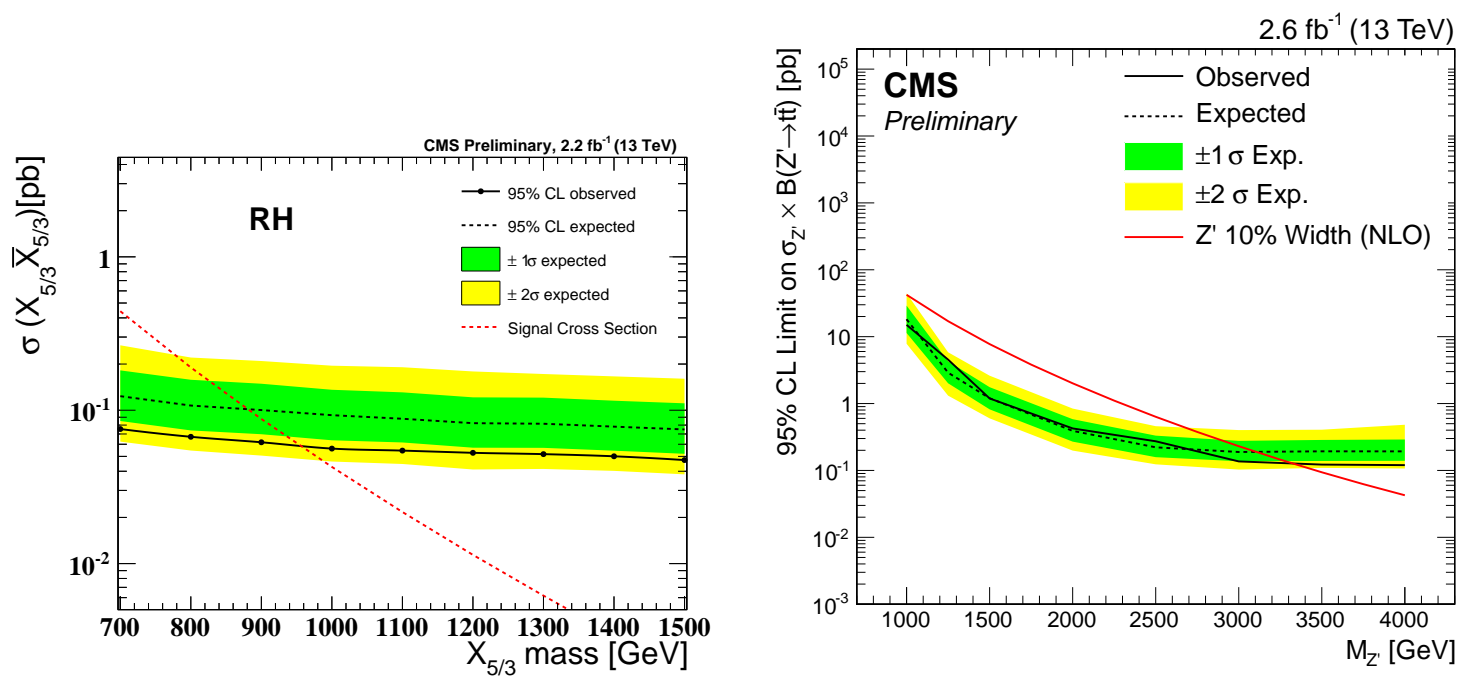

Figure 5: Left: expected and observed 95\% confidence level upper limits on the production cross section of a right-handed top quark partner $X^{5 / 3}$ [7]. Right: expected and observed $95 \%$ confidence level upper limits on the cross section times branching ratio for a $10 \%$ width $Z$ boson as a function of the mass [8].

Also the hunt to discover signatures from theories beyond the standard model involving supersymmetry is very much alive. Summary plots with exclusion limits for various simplified models obtained from a wide range of searches can be found in Ref. [9], while two examples are presented in Figure 6. The left panel shows the exclusion limits for gluino pair production, with the gluino decaying to a top quark pair and a neutralino as Lightest Supersymmetric Particle (LSP). The exclusion limits are presented as $95 \%$ confidence level bounds in the plane of the mass of the gluino and the neutralino. We observe a huge jump in sensitivity with respect to the Run-1 limits. In particular, for a massless neutralino, the mass-limit for the existence of a gluino is at least $200 \mathrm{GeV}$ higher. Similarly, the right panel shows the exclusion limits for direct production of a stop quark 
pair, with the stop quark decaying to a top quark and a neutralino as LSP. In this case the exclusion limits are presented as $95 \%$ confidence level bounds in the plane of the mass of the stop quark and the neutralino. Also in this case, multiple results are obtained using the $13 \mathrm{TeV}$ proton collision data. The region where the stop quark mass is close to the sum of the top quark and neutralino masses is particularly hard to exclude due to the very similar kinematics of stop quark pair production and top quark pair production. Apart from dedicated searches, this so-called stealth-susy region is probed through precision measurements of the top quark pair production cross section.
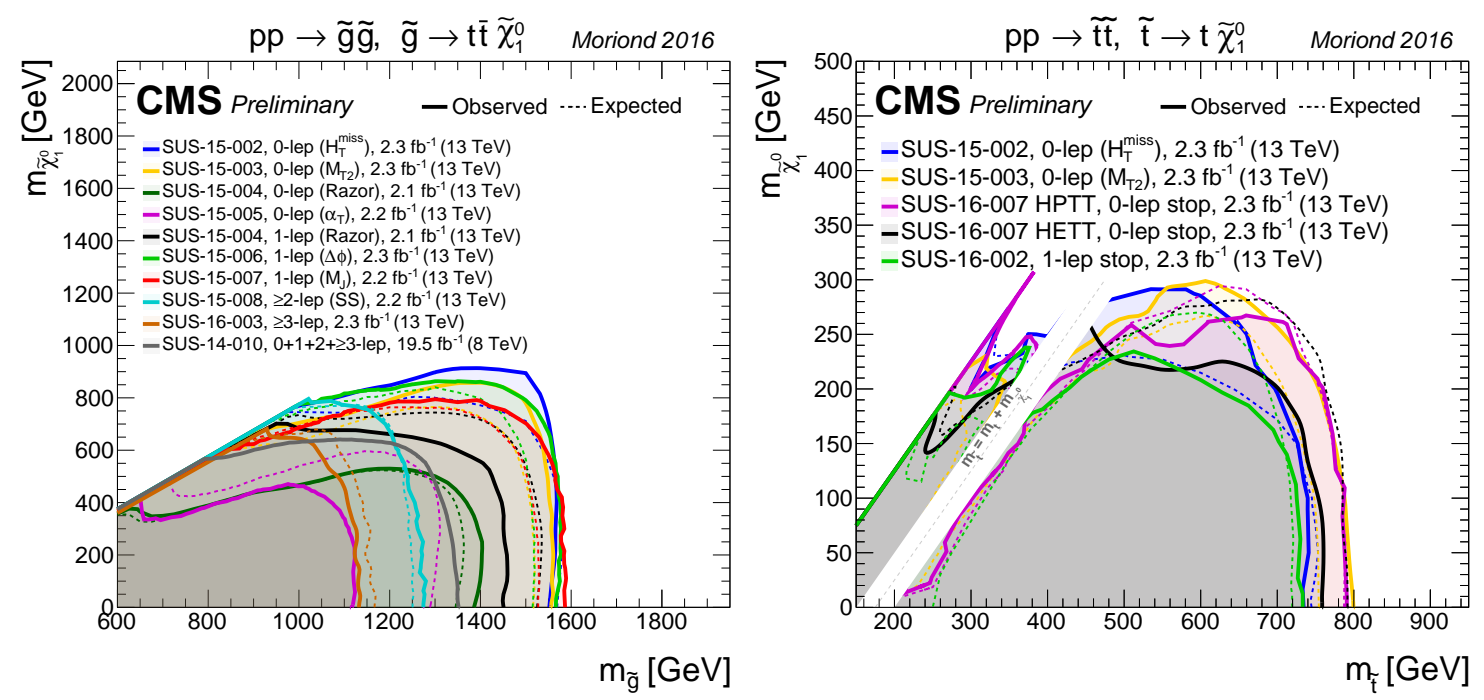

Figure 6: Left: expected and observed 95\% confidence level upper limits on the production cross section of gluino pair production, with the gluino decaying to a top quark pair and a neutralino [9]. The limits are presented in the plane of the the mass of the gluino and the neutralino. Right: expected and observed $95 \%$ confidence level upper limits on the production cross section of stop quark pair production, with the stop quark decaying to a top quark pair and a neutralino. The limits are presented in the plane of the the mass of the stop quark and the neutralino [9].

Apart from the popular standard model extensions, there is a rich variety of more exotic theories beyond the standard model which can manifest themselves in a very diverse number of signatures. Towards the end of 2015, a lot of attention was triggered due to an excess observed in the diphoton invariant mass distribution. The CMS collaboration reanalyzed the data, also including the proton collision data recorded when the magnetic field was off. The results are presented in $[10,11]$. Figure 7 shows the diphoton invariant mass distribution when the two photons are in the barrel. The left (right) panel relies on the data with a magnetic field of 3.8 (0) T. Around a diphoton invariant mass of $750 \mathrm{GeV}$ an excess of events is observed with respect to the rapidly falling background. To quantify the importance of this excess, one can calculate the exclusion limits and the significance (or background-only p-value) combining the proton collision data collected at 8 and $13 \mathrm{TeV}$. The exclusion limits are presented in Figure 8 and the background-only p-values in Figure 9. The largest excess has a local significance of $3.4 \sigma$ at a diphoton invariant mass of 750 $\mathrm{GeV}$. Clearly, this puzzling result triggered many other searches for a scalar particle with a mass around $750 \mathrm{GeV}$. One example is the search for a scalar resonance decaying to a $Z$ boson and a photon [12]. The upper limit on the production cross section of such a particle is shown in the left 

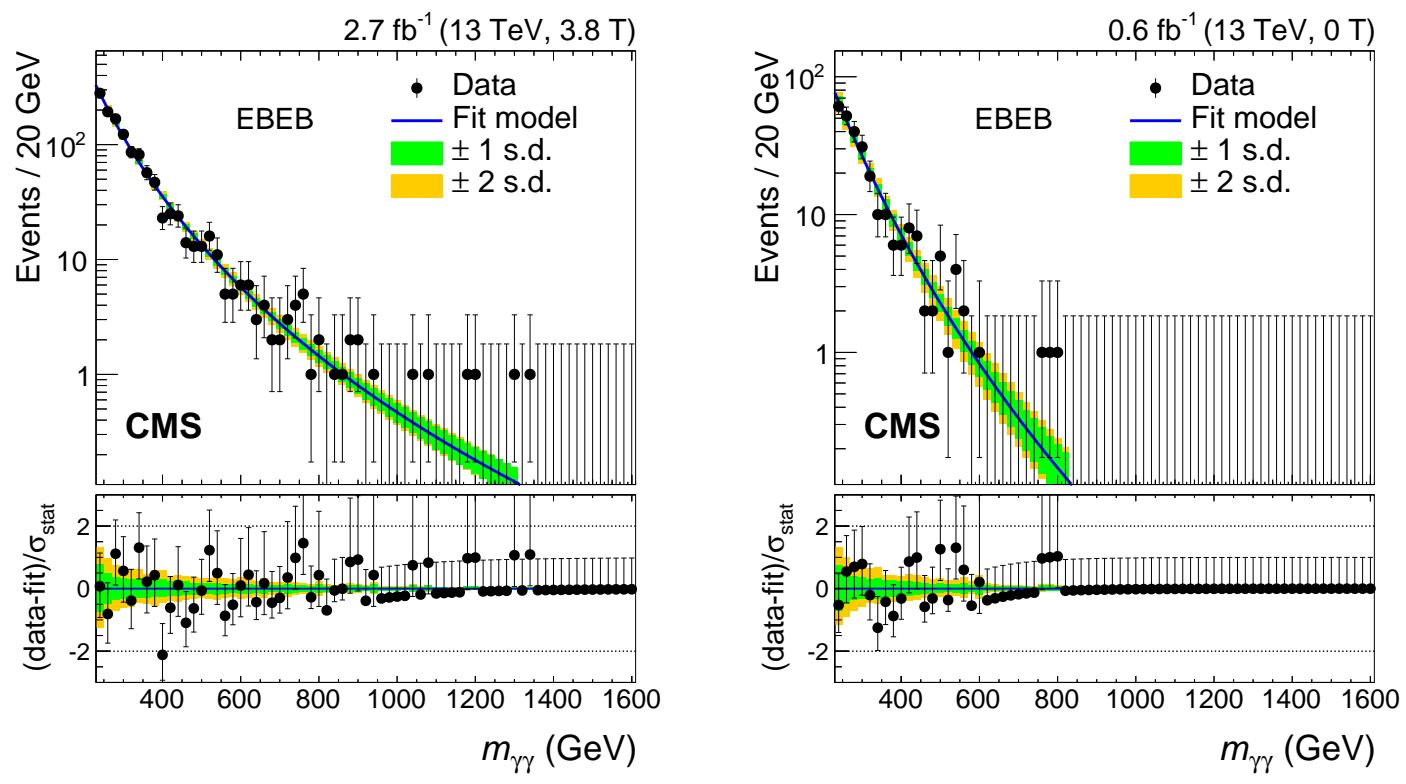

Figure 7: Left: distribution of the diphoton invariant mass for events recorded when the magnetic field at the nominal value of 3.8 T. Right: distribution of the diphoton invariant mass for events recorded when there was no magnetic field. In both cases one can see an excess of events around a diphoton invariant mass of $750 \mathrm{GeV}[10,11]$.

panel of Figure 10, while the background-only p-values of the corresponding search is shown in the right panel. In this case the largest excess with an $Z \gamma$ invariant mass around $370 \mathrm{GeV}$ corresponds to a local significance of $2.6 \sigma$.

It is worth nothing that when more data was included after the LHCP conference, the significance of the excess in the diphoton invariant mass distribution was reduced [13].

\subsection{Heavy flavour physics}

New physics effects can also be indirectly observed for instance when studying $B$ hadron production and decays. The advantage is that these measurements are able to probe the scale of new physics. A measurement of the $B^{+}$meson differential production cross section was performed as a function of the transverse momentum and rapidity. The measurement uses the exclusive decay channel $B^{+} \rightarrow J \backslash \psi K^{+}$, with the $J \backslash \psi$ decaying to a pair of muons [14]. As can be seen from the left panel of Figure 11, the results agree reasonably with theory calculations and are also compared with measurements made at a center of mass energy of $7 \mathrm{TeV}$.

Also the differential prompt production cross sections of quarkonium was measured using the $13 \mathrm{TeV}$ proton collision data. The five $\mathrm{S}$-wave quarkonium states were reconstructed in the dimuon decay channel, for dimuon rapidity $|y|<1.2$ [15]. An example is shown in the right panel of Figure 11 for the $\Upsilon(n S)$ quarkonium states. The double differential $13 \mathrm{TeV}$ cross section of the quarkonium states is two to three times larger than at $7 \mathrm{TeV}$ following the expectation due to the evolution of the parton distribution functions. As was the case for the Run-1 results, no significant deviations are observed for the heavy flavour physics measurements. 


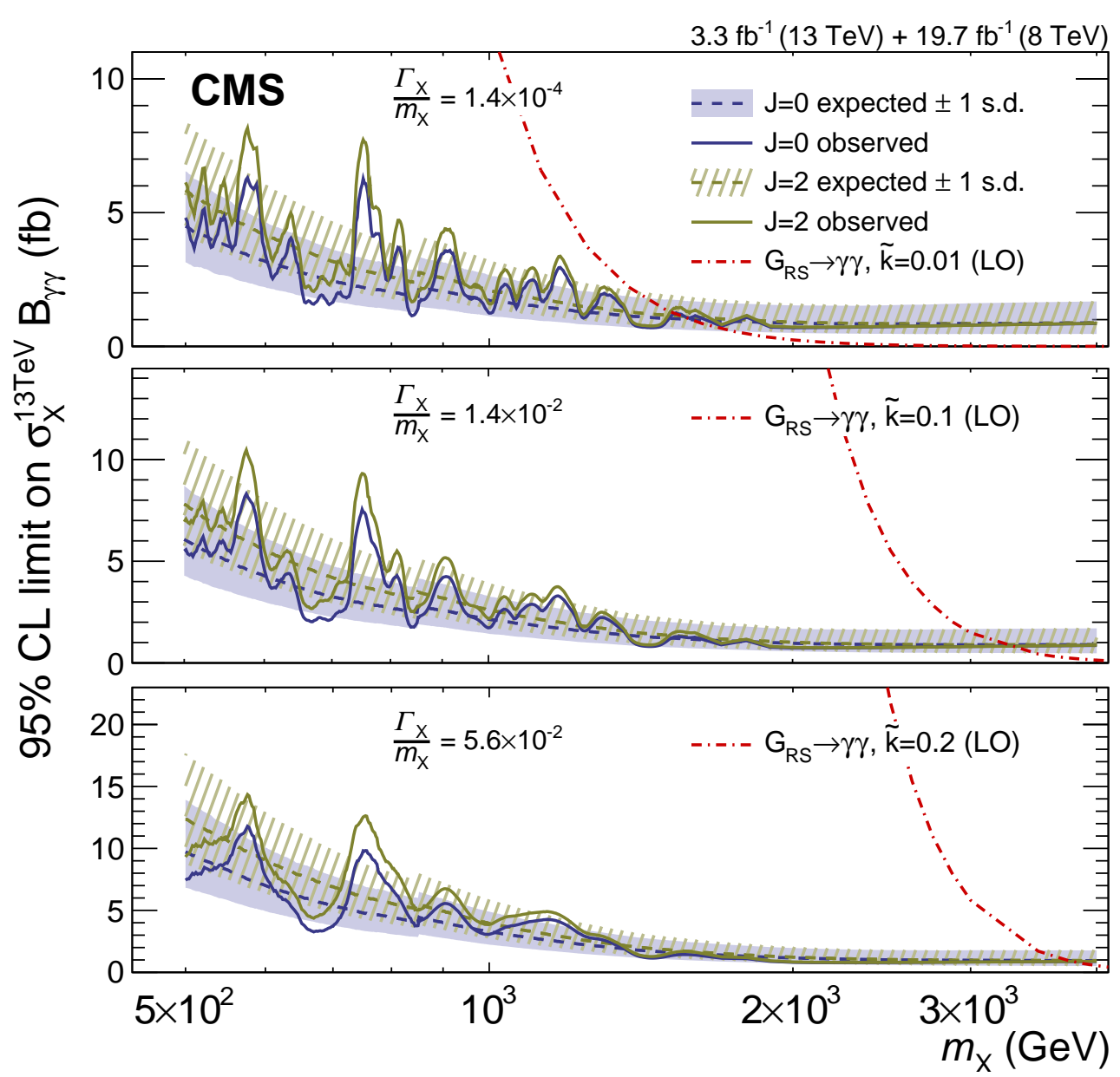

Figure 8: The $95 \%$ confidence level upper limits on the production of diphoton resonances as a function of the resonance mass $m_{X}$, from the combined analysis of the 8 and $13 \mathrm{TeV}$ data. The $8 \mathrm{TeV}$ results are scaled by the ratio of the 8 to $13 \mathrm{TeV}$ cross sections. The blue-grey (darker) curves and the green (lighter) ones correspond to the scalar and RS graviton signals, respectively. Solid (dashed) curves represent the observed (median expected) exclusion limit. The expected results are shown with their 1 standard deviation dispersion bands. The leading-order RS graviton production cross section is shown by the red dot-dashed curves. The results are shown for (upper) a narrow, (middle) an intermediate-width, and (lower) a broad resonance, with the value of the width $\Gamma_{X} / m_{X}$, relative to the mass, indicated in the legend of each plot [10, 11].

\subsection{Physics in heavy ion collisions}

Based on the 2015 collision data, interesting advances were also made on the topic of heavy ion collisions. Only two results are highlighted since it is impossible to listing all the new measurements.

The nuclear modification factor $R_{A A}$ was measured for prompt $D^{0}$ production [16]. It is defined as

$$
R_{A A}\left(p_{T}\right)=\frac{1}{T_{A A}} \frac{d N_{P b P b}^{D^{0}}}{d p_{T}} / \frac{d \sigma^{D^{0}} p p}{d p_{T}}
$$




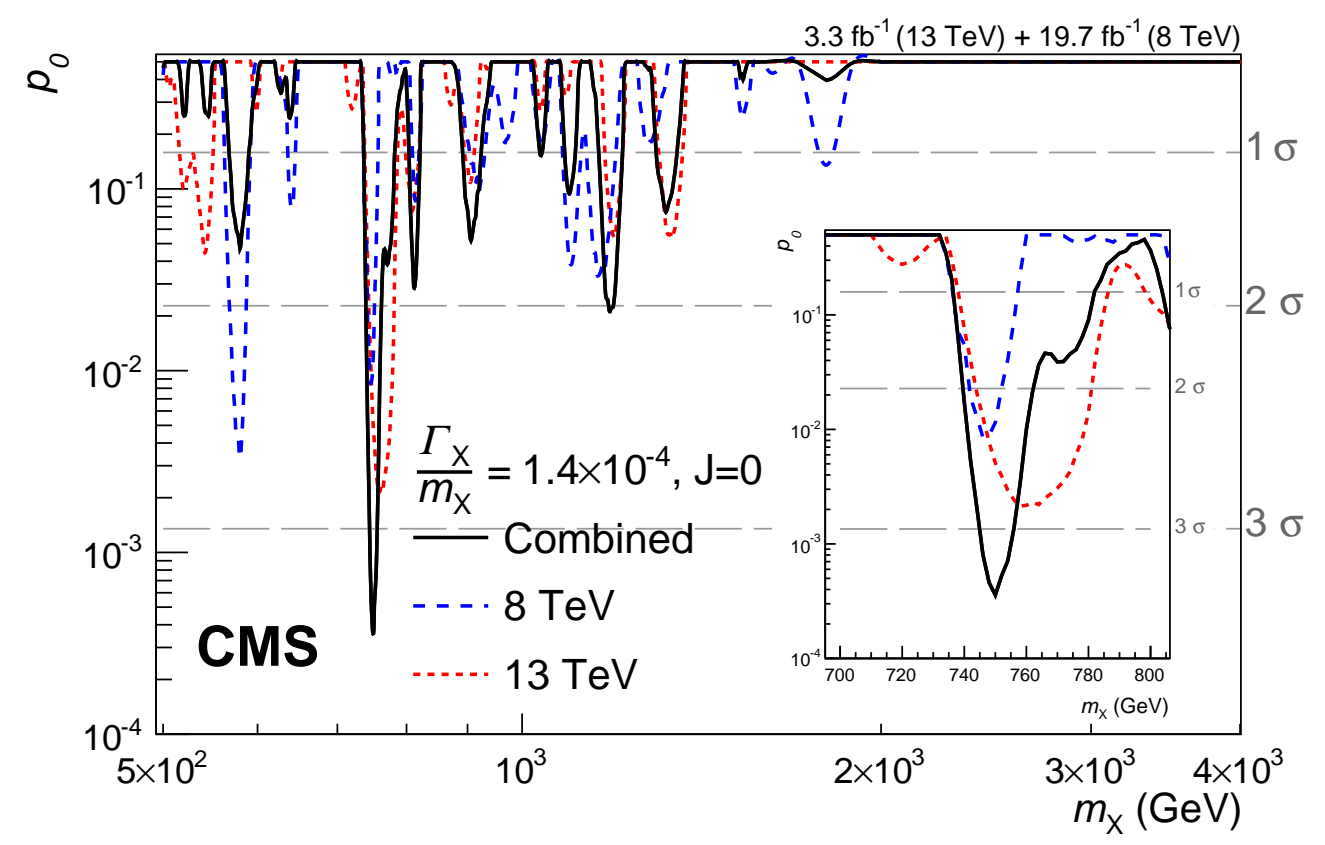

Figure 9: Observed background-only p-values for narrow-width scalar resonances as a function of the resonance mass $m_{X}$, from the combined analysis of the 8 and $13 \mathrm{TeV}$ data. The results for the separate 8 and $13 \mathrm{TeV}$ data sets are also shown. The inset shows an expanded region around $m_{X}=750 \mathrm{GeV}[10,11]$.
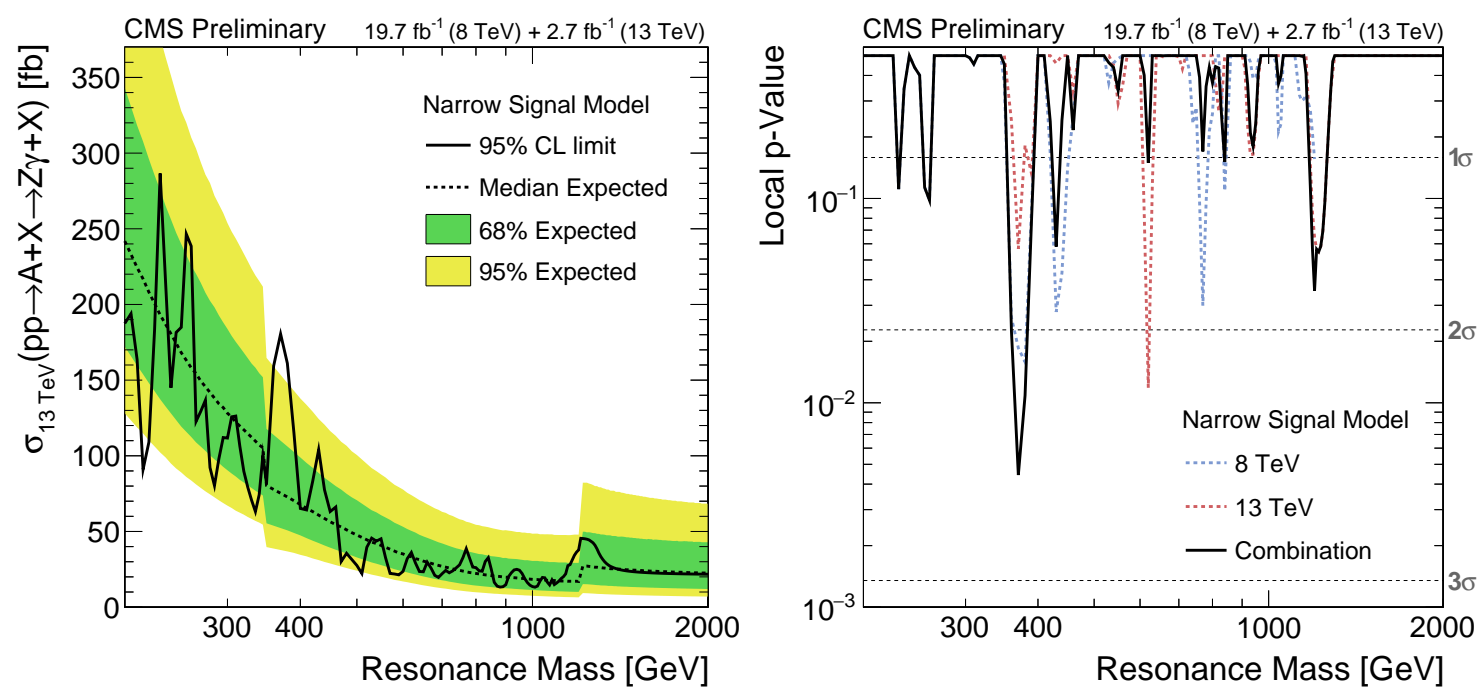

Figure 10: Left: combined 8 and $13 \mathrm{TeV}$ expected and observed 95\% confidence level upper limits on the production cross section of a narrow width spin- 0 resonance decaying to $Z \gamma$. The discontinuities arise from the combination of the $8 \mathrm{TeV}$ and $13 \mathrm{TeV}$ results. Right: background-only p-value as a function of the $Z \gamma$ invariant mass for the $8 \mathrm{TeV}$ and $13 \mathrm{TeV}$ results separately and for their combination [12]. 

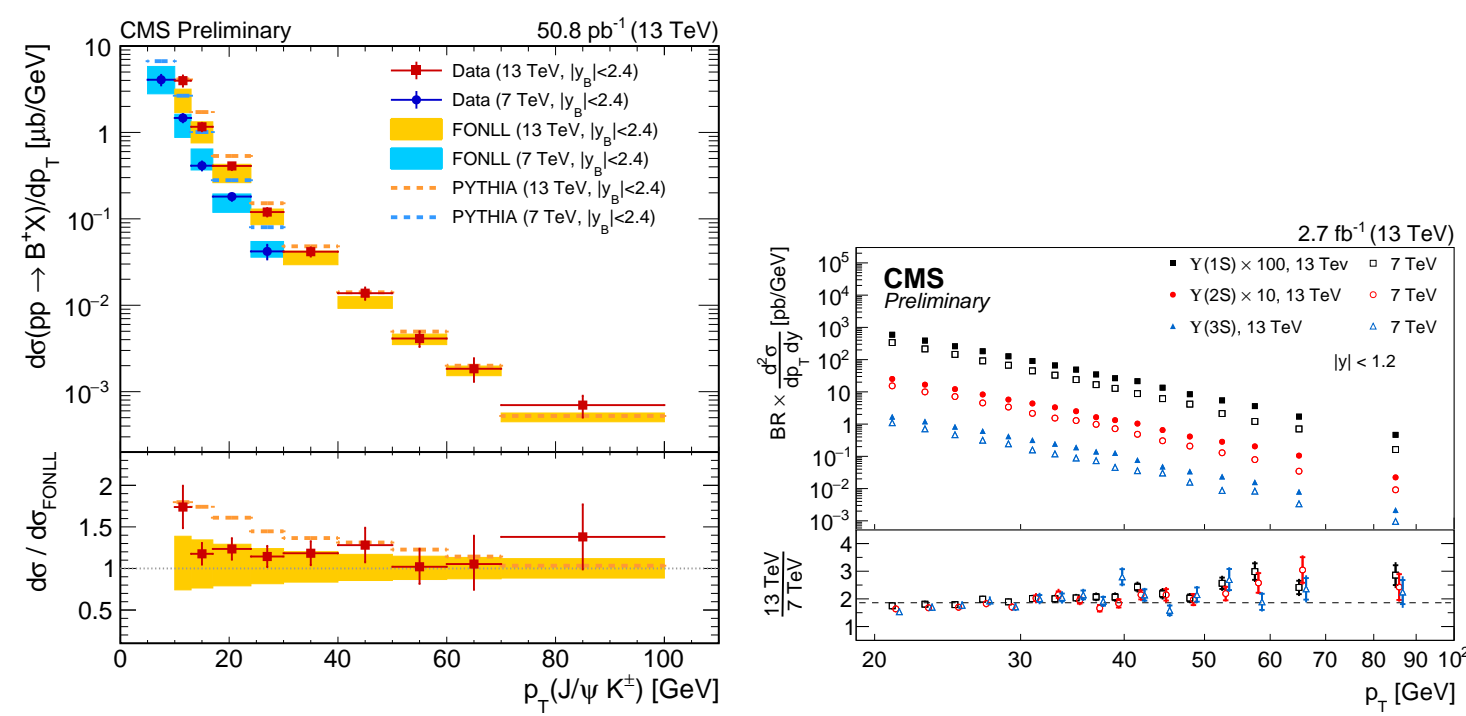

Figure 11: Left: Differential production cross sections for $B^{+}$production as a function of the transverse momentum for a rapidity $|y|<2.4$ [14]. Right: Differential cross sections times branching ratios for $7 \mathrm{TeV}$ and $13 \mathrm{TeV}$ data, for $\Upsilon(n S)$, for $|y|<1.2$, assuming isotropic dimuon decays [15].

with $T_{A A}$ the nuclear overlap function. Hence, it is the ratio between the corrected lead-lead yield and the proton-proton cross section scaled by the number of incoherent nucleon-nucleon collisions. For a centrality range between 0 and 10\%, there is a suppression of a factor five between 6 and 7 $\mathrm{GeV}$ and a factor 1.5 between 60 and $100 \mathrm{GeV}$. The measured $D^{0}$ nuclear modification factor is compatible with the inclusive charged hadron nuclear modification factor, within the experimental uncertainties.

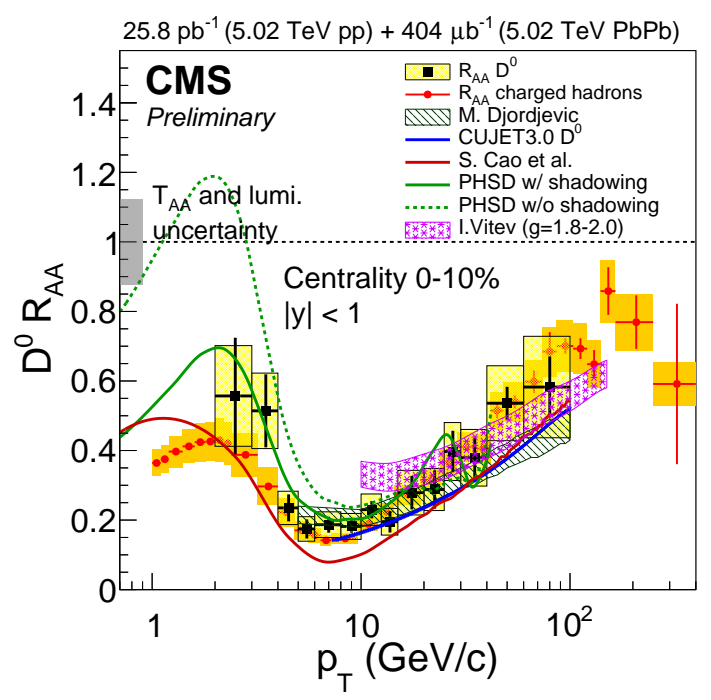

Figure 12: The nuclear modification factor $R_{A A}$ for prompt $D^{0}$ mesons as a function of the transverse momentum in the centrality range $0-10 \%$. The inclusive charged particle $R_{A A}$ results are superimposed for an equivalent event selection and compared to the predicted $D^{0} R_{A A}$ from various theoretical calculations [16]. 
For flow studies, the azimuthal asymmetry of the final state spectra is measured. The azimuthal part of the momentum distribution of hadrons is usually decomposed into a Fourier series to obtain

$$
\frac{d N}{d \phi} \propto 1+2 v_{2} \cos \left(2\left(\phi-\phi_{0}\right)\right)+\cdots
$$

The second order harmonic $v_{2}$ is related to the so-called elliptic flow and was measured for twoand multi-particle angular correlations in proton-proton collisions at center of mass energies of 5,7 and $13 \mathrm{TeV}$ as a function of charged-particle multiplicity [17]. The results are presented in Figure 13 for proton-proton collisions (left panel), proton-lead collisions (middle panel) and leadlead collisions (right panel). The measurement for four- and six-particle correlations is similar to that of two-particle correlations in the three cases. These results are particularly important since the observation provides evidence for the collective nature of long-range correlations in highmultiplicity proton-proton events.

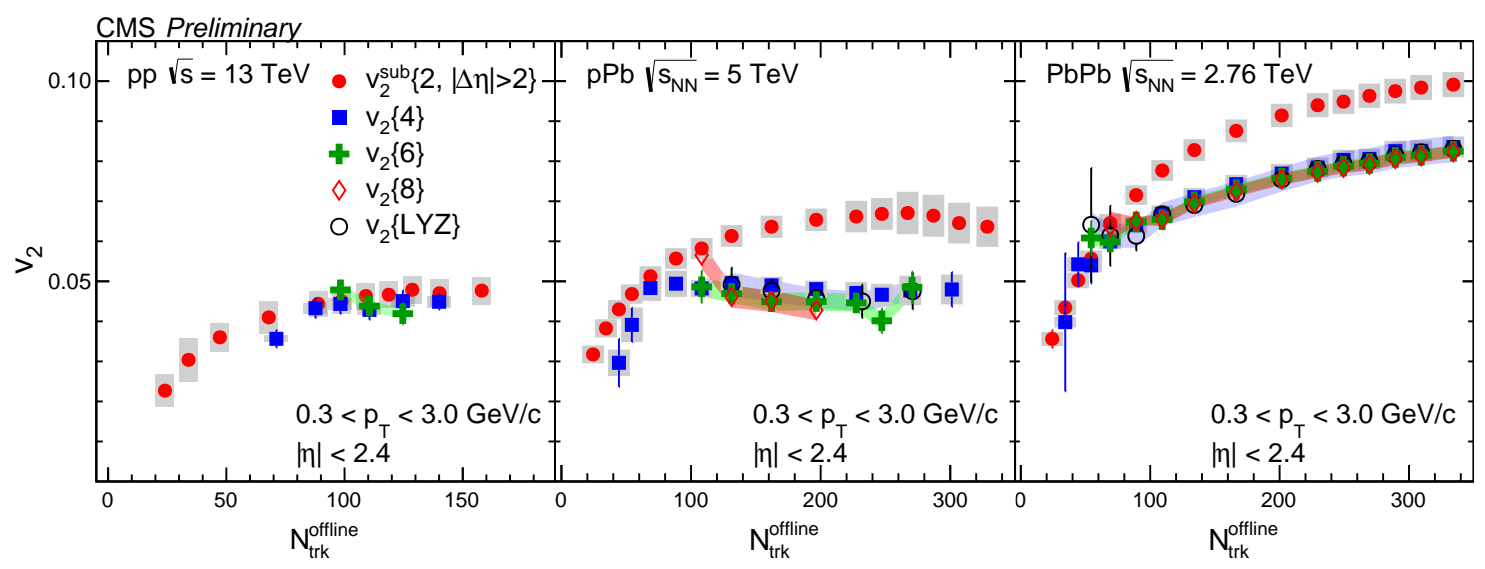

Figure 13: Left: The $v_{2}^{\text {sub }}, v_{2}\{4\}$ and $v_{2}\{6\}$ values as a function of $N_{\text {trk }}^{\text {offline }}$ for charged particles, averaged over $0.3<p_{T}<3.0 \mathrm{GeV}$ and $|\eta|<2.4$, in pp collisions at $\sqrt{s}=13 \mathrm{TeV}$. Middle: The $v_{2}^{\text {sub }}, v_{2}\{4\}, v_{2}\{6\}$, $v_{2}\{8\}$, and $v_{2}\{\mathrm{LYZ}\}$ values in $\mathrm{pPb}$ collisions at $\sqrt{s_{N N}}=5 \mathrm{TeV}$. Right: The $v_{2}^{\text {sub }}, v_{2}\{4\}, v_{2}\{6\}, v_{2}\{8\}$, and $v_{2}\{\mathrm{LYZ}\}$ values in $\mathrm{PbPb}$ collisions at $\sqrt{s_{N N}}=2.76 \mathrm{TeV}$ [17].

\section{Overall status of the CMS experiment at the start of 2016}

At the start of the LHC Run-2 in 2015, it became clear that there were issues with the cryogenic system of the CMS solenoid. As a result the solenoid could not be operated stably and part of the proton collision data was collected without magnetic field. During the technical stop and before resuming the collisions in 2016 , an intense programme was followed to fully refurbish the cryogenic system for stable operation with a magnetic field of $3.8 \mathrm{~T}$. In particular, the cold box of the cryogenic system was carefully cleaned to remove traces of the Breox (oil) contiminant. Secondly, the primary oil removal system was fully replaced. A magnetic field at the nominal value of 3.8 T was reached on the 28th of April 2016 and the cryogenic system was operated stably ever since. We would like to explicitly thank our colleagues from the CERN-TE dept, the technical support from other CERN departments, CERN-EN, EP, the CERN Management, the CMS Magnet 
team and integration office, contractors (particularly Altead, ZEC service) and CMS members for support and advice during this critical period!

With the excellent performance of the LHC accelerator also in 2016, the CMS experiment was able to efficiently collect proton collision data corresponding to an integrated luminosity of almost $3 \mathrm{fb}^{-1}$ on a time scale of a couple of weeks. During this period, the performance of each of the subdetectors was excellent as can be seen from Figure 14 showing the fraction of active channels for each of the subdetectors. The fraction of active subdetector channels at the start of the 2016 run is similarly as at the end of 2015 and over $95 \%$ for all subdetectors [18]. For the preshower the fraction of active channels is actually $3 \%$ better than at the end of 2015 since a number of channels was recovered after on-detector repairs.

\section{Detector Active Fraction}

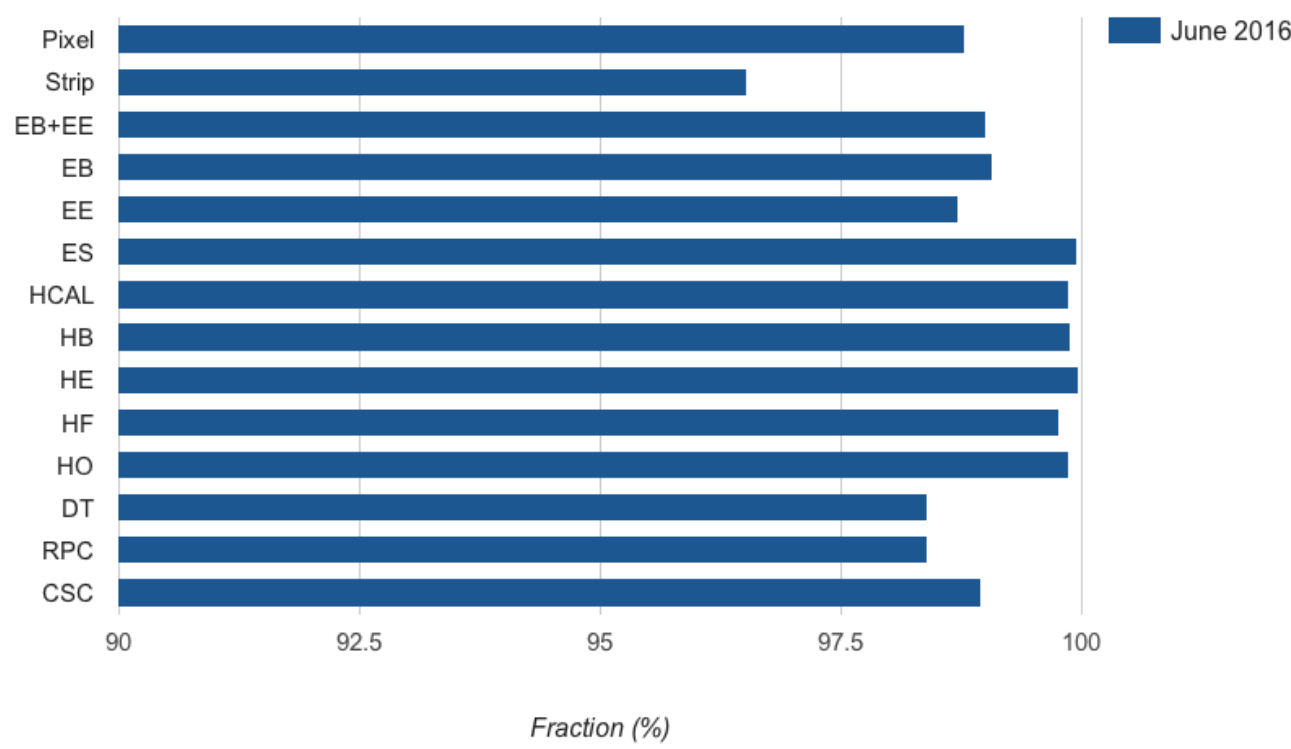

Figure 14: Fraction of active CMS subdetector channels in June 2016, where you can appreciate the $x$-axis starting at $90 \%$ [18].

\section{Object readiness and performance at the start of 2016}

In 2016 the LHC will reach an instantaneous luminosity exceeding $10^{34} \mathrm{~cm}^{2} \mathrm{~s}^{1}$ and an average number of pile-up interactions of at least 40. The CMS Level-1 (L1) trigger architecture has undergone a full upgrade in order to maintain and improve the trigger performance under these extreme conditions. In this way, we can keep the trigger rate under control and avoid a significant increase of the trigger thresholds.

The left panel in Figure 15 shows the difference between the L1 and offline jet transverse energy for jets in the hadronic calorimeter barrel. Offline jets are reconstructed with the Particle Flow algorithm and dedicated calibrations are applied to the L1 jets. The distribution is nicely centered around 0 and the dependency on the number of pile up collisions is relatively small. For 
jets in the forward calorimeter, there is a small dependency on the number of pile up collisions, but it does not impact the performance significantly [19].

Before 2016, the muon track finders for the L1 trigger were detector based, while today they are based on pseudorapidity (endcap, barrel and overlap region) to exploit the detector redundancy already at a lower level. A global muon trigger takes care of merging and removing duplicate trigger objects [20]. The right panel in figure 15 shows the efficiency of the SingleMuon L1 trigger as a function of the reconstructed muon transverse momentum. The efficiency is measured with a tag and probe method in $Z \rightarrow \mu \mu$ events and the reconstructed muons are required to have a transverse momentum exceeding $22 \mathrm{GeV}$. For muons with a transverse momentum above $20 \mathrm{GeV}$ the efficiency is around $93 \%$.
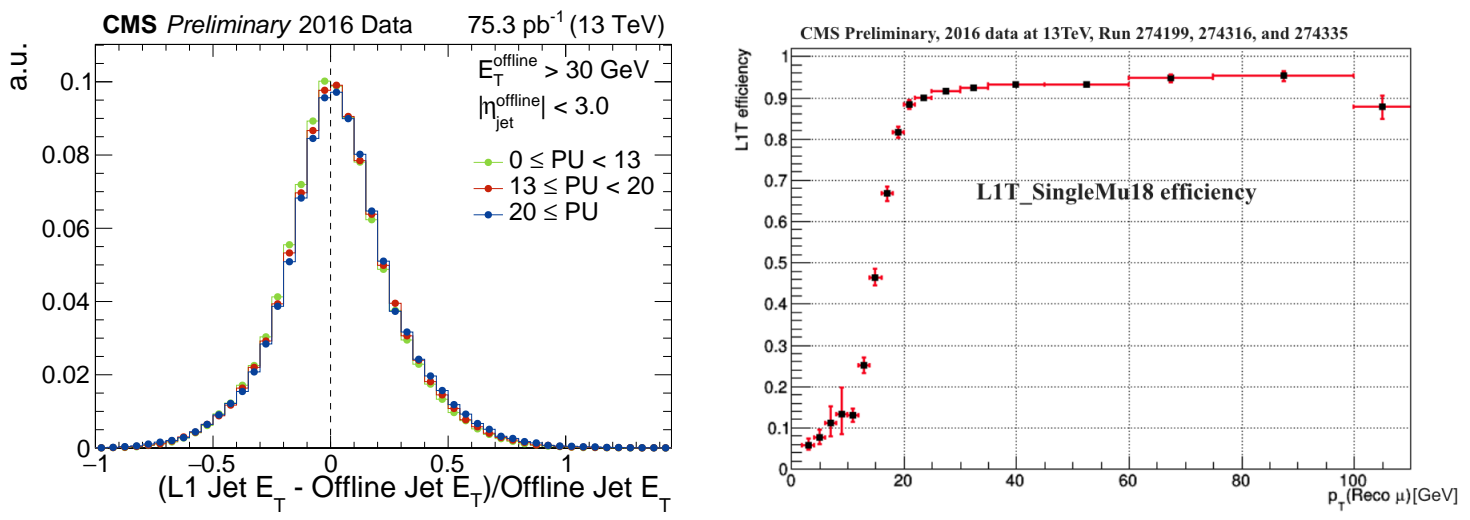

Figure 15: Left: jet transverse energy resolution [19]. Right: efficiency of the SingleMuon L1 trigger as a function of the reconstructed muon transverse momentum measured in $Z \rightarrow \mu \mu$ events. The highest $p_{T}$-bin includes the overflow [20].

Important for many heavy flavour physics analyses are the low-mass dimuon resonances. The dimuon invariant mass distribution shown in the left panel of Figure 16 shows our ability to reconstruct the full spectrum with the dimuon resonances at the expected mass values [21]. The right panel shows the efficiency to find an isolated muon as a function of its transverse momentum. The efficiency is close to unity for muons passing the tight identification requirements and a transverse momentum exceeding $60 \mathrm{GeV}$ [22].

The performance of the electromagnetic calorimeter (ECAL) can be illustrated with Figure 17 [23]. In the left panel the invariant mass of photon pairs is shown in the $\pi^{0}$ mass range with one photon in a certain crystal of the ECAL barrel. These events are selected with a dedicated trigger path and used as prompt feedback to monitor the effectiveness of the laser monitoring calibration and to intercalibrate the energy of ECAL crystals. The root mean square on the relative energy scale during a fill is around 7\% when this calibration is applied.

The right panel of Figure 17 shows the residual difference in $\eta$ between the position of the ECAL supercluster and the tracker based position in the ECAL endcaps. A relative ECAL-tracker alignment precision of $1(2) \times 10^{3}$ units in $\eta$ is obtained in the ECAL barrel (endcaps). This meets the ECAL alignment goals for electron identification and diphoton resonance reconstruction, which are $4 \times 10^{3}$ units in $\eta$. A similar conclusion is made for $\phi$ [23]. 

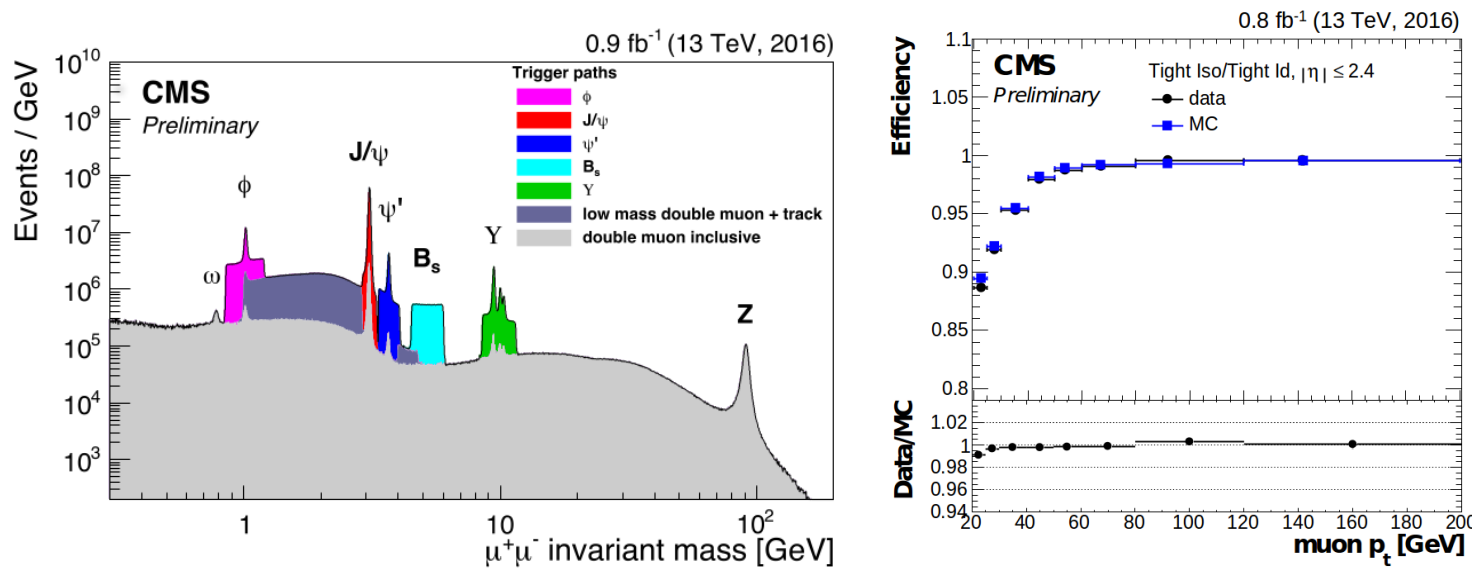

Figure 16: Left: Dimuon invariant mass distribution collected with various dimuon triggers. The light gray continuous distribution represents events collected with inclusive dimuon triggers with high $p_{T}$ thresholds. The dark gray band is collected by a trigger with low-mass non-resonant dimuon plus a track. The other colored spectra are acquired using specialized triggers which require a pair of muons with opposite charge, a vertex-fit probability greater than $0.5 \%$, and specific dimuon invariant mass and $p_{T}$ regions [21]. Right: efficiency for identifying an isolated muon as a function of the muon transverse momentum. Muons from $Z \rightarrow \mu \mu$ events are selected by applying the tight identification requirements and requiring tight isolation criteria [22].
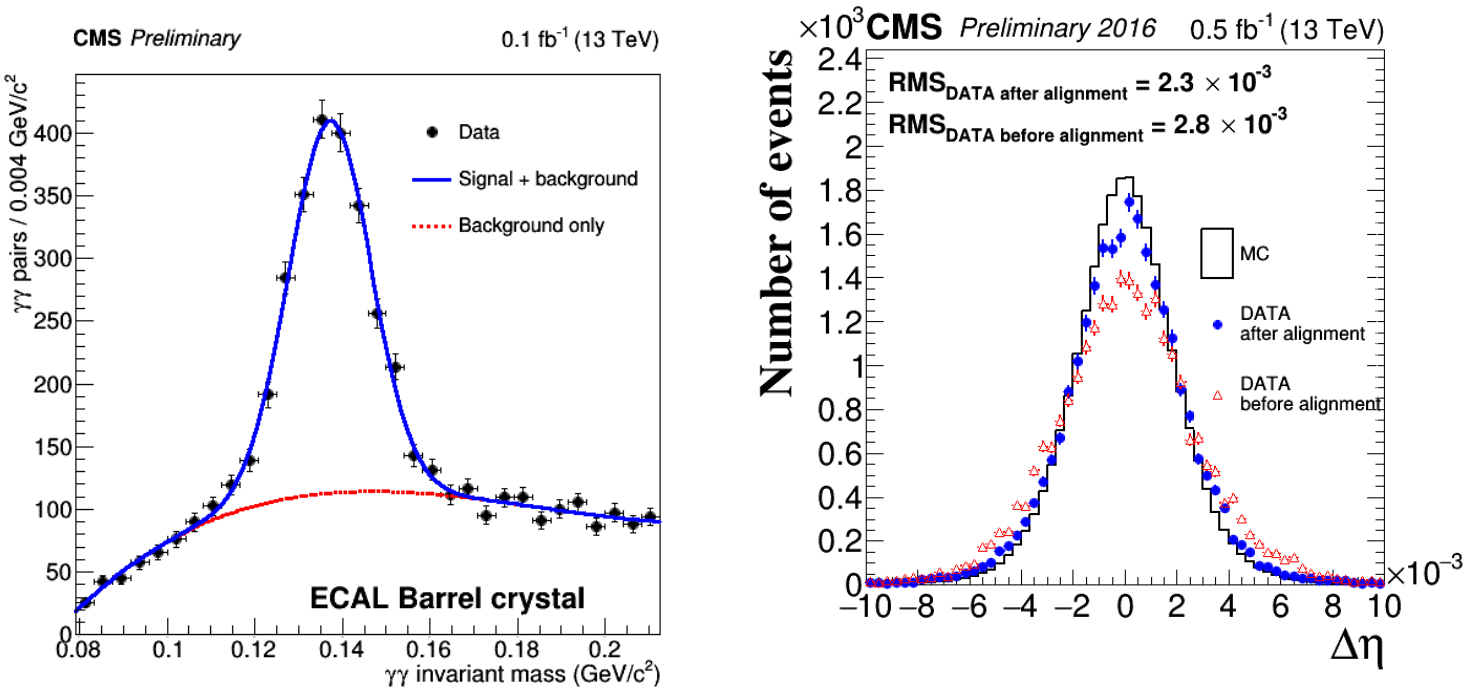

Figure 17: Left: the invariant mass of photon pairs showing the $\pi^{0}$ mass peak with one photon in a fixed crystal of the ECAL barrel, at $|\eta|=0.5$. Right: The distribution of $\Delta \eta=\eta_{\text {ECALsupercluster }}-\eta_{\text {tracker }}$ using $Z \rightarrow e e$ events for data using the 2015 ECAL alignment and after the 2016 ECAL alignment procedure has been carried out. The distributions are shown for the ECAL endcaps and after applying the 2016 ECAL alignment correction it agrees reasonably well with the perfectly aligned simulated events [23]. 
The jet energy response is measured in simulated proton collisions and in data using $p_{T}$ balancing in $\gamma+$ jet, $Z \rightarrow \mu \mu+$ jet and $Z \rightarrow e e+$ jet events. The ratio of the data to simulated jet energy response is shown in the left panel of Figure 18 after setting the nuisance parameters to their fitted values. The kink observed around $150 \mathrm{GeV}$ is attributed to changes in local reconstruction in the hadronic calorimeter between Run-1 and Run-2. The scale at low jet transverse momentum is around 0.98 , consistent with Run- 1 results, and evolves towards 1 at higher jet transverse momenta [24].
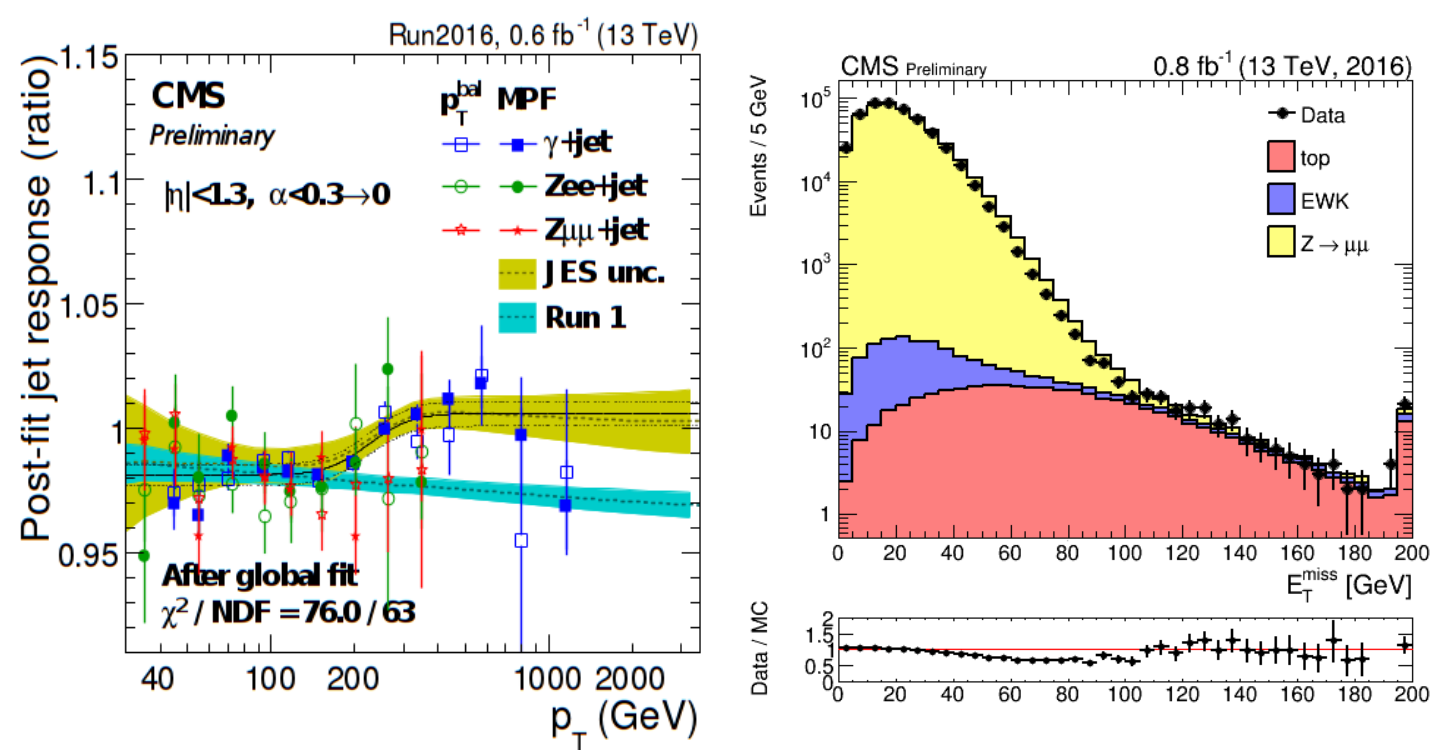

Figure 18: Left: post-fit jet response as a function of the jet transverse momentum at $13 \mathrm{TeV}$ and fitted with a function $a+b\left(\operatorname{lerf}\left(\left(\log \left(p_{T}\right)-\log (225)\right) / 0.4\right)\right)$ [24]. Right: Missing transverse energy in $Z \rightarrow \mu \mu$ events at $13 \mathrm{TeV}$ [25].

The scale and resolution of the missing transverse energy can be measured in $\gamma+$ jet and $Z \rightarrow l l$ events by comparing the transverse momentum of the well measured vector boson to that of the hadronic recoil system. The missing transverse energy obtained with particle flow is corrected for the jet energy scale. The right panel in Figure 18 shows the distribution of the missing transverse energy for events passing a $Z \rightarrow \mu \mu$ selection [25]. It can be seen that the distribution is quite well modelled, especially when taking into account that the residual jet energy corrections are not applied.

Also the high-level object identification algorithms have been reoptimized for Run-2. An example is the identification of hadronic $\tau$ decays as shown in the left panel of Figure 19. The improved MVA-based identification efficiency is performing significantly better than the cut-based strategy, with a $20 \%$ difference in relative efficiency [26].

For $b$ jet identification the CombinedSecondaryVertex (CSV) algorithm was significantly improved and a new algorithm combinedMVA (cMVA) was developed that combines the other algorithms using a Boosted Decision Tree [27]. The ROC curves illustrating the performance of the various $b$ jet identification algorithms are shown in the right panel of Figure 19 as well as a comparison with the Run-1 CSV algorithm. 

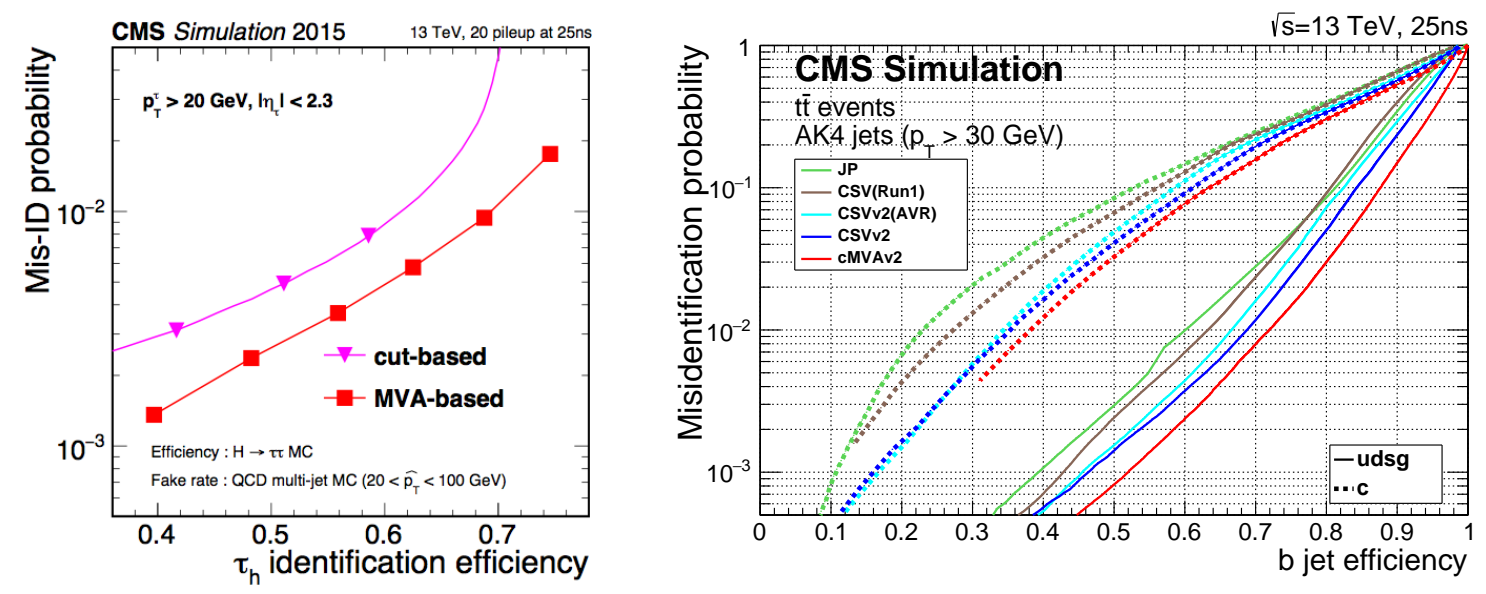

Figure 19: Left: misidentification probability as a function of the hadronic $\tau$ identification efficiency in multijet events (for the background) and Higgs decays to two $\tau$ leptons (for the signal) at $13 \mathrm{TeV}$ [26]. Right: misidentification probability for $c$ and $d, u, s, g$ jets as a function of the $b$ jet identification efficiency in $t \bar{t}$ events at $13 \mathrm{TeV}$ [27].

\section{Conclusion and outlook}

We discussed a number of physics results based on the Run- 2 collision data. In addition, we also illustrated the performance of the detector and the object reconstruction performance at the start of 2016. The CMS collaboration is also active in many other areas, such as for instance the upgrade of the silicon pixel tracker for 2017 and the upgraded CMS detector for the HL-LHC, topics that were not discussed here. It should also be noted that we are only at the beginning of analyzing the vast amount of data to be collected for Run- 2 and we will collect a dataset in 2016 that brings unique opportunities both concerning searches for new physics phenomena and for precision measurements. This is illustrated in Figure 20, where the ratio of the predicted cross section at 13 $\mathrm{TeV}$ to that at $8 \mathrm{TeV}$ is shown for a number of benchmark processes. With the integrated luminosity collected at the end of 2016, we will be able to probe all of these processes in detail and a discovery may be just around the corner!

\section{References}

[1] CMS Collaboration, The CMS experiment at the CERN LHC, JINST 33 S08004 (2008).

[2] CMS Collaboration, Measurement of the double-differential inclusive jet cross section at $\sqrt{s}=13$ TeV, Eur. Phys. J. C 76 (2016) 451 [hep-ex/1605.04436].

[3] CMS Collaboration, Measurement of inclusive $W$ and $Z$ boson production cross sections in $p p$ collisions at sqrts $=13 \mathrm{TeV}$, CMS-PAS-SMP-15-004.

[4] CMS Collaboration, LHC Top Working Group Summary Plots, https://twiki.cern.ch/twiki/bin/view/LHCPhysics/LHCTopWGSummaryPlots.

[5] CMS Collaboration, Studies of Higgs boson production in the four-lepton final state at $\sqrt{s}=13 \mathrm{TeV}$, CMS-PAS-HIG-15-004. 


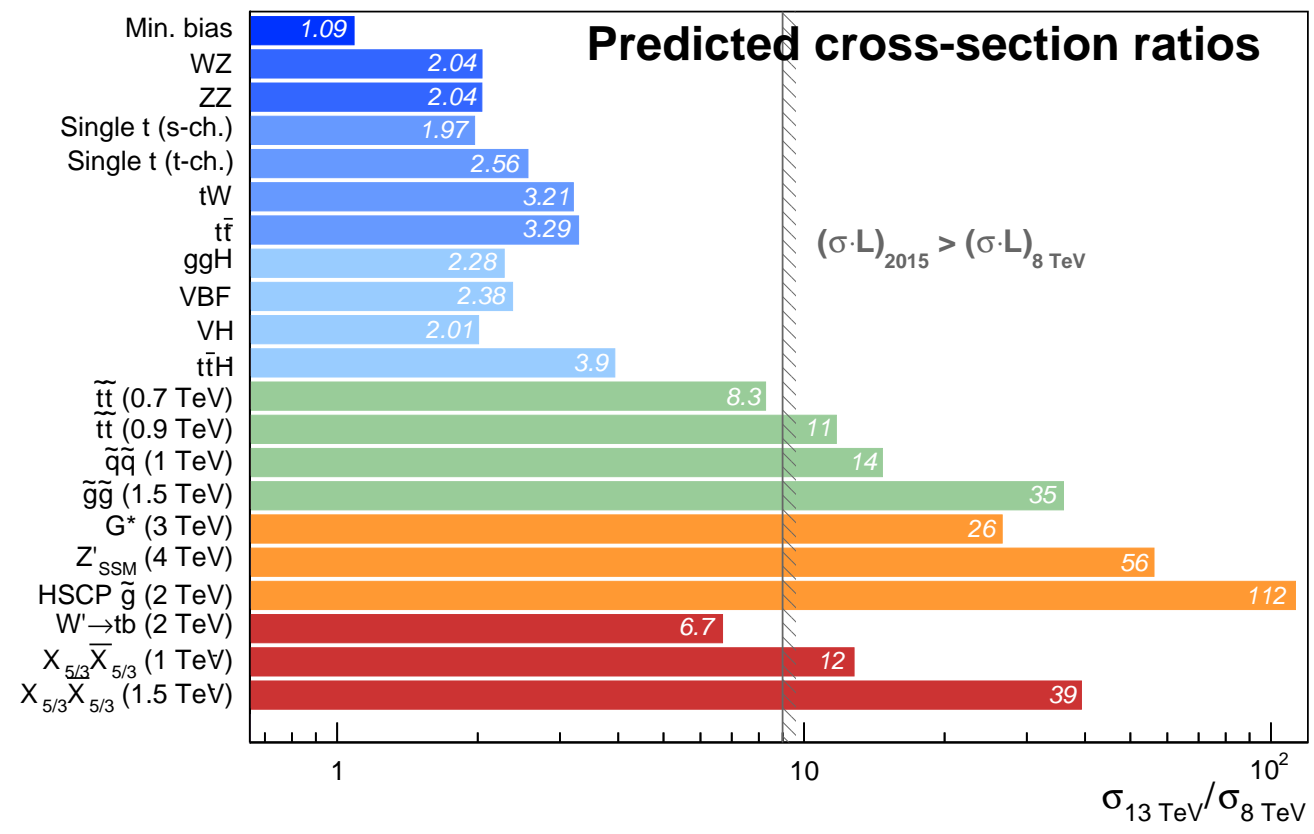

Figure 20: Ratio of the predicted cross section at a center of mass energy of $13 \mathrm{TeV}$ and $8 \mathrm{TeV}$. The line indicates where the number of events collected in 2015 exceeds the number of events collected at a center of mass energy at $8 \mathrm{TeV}$.

[6] CMS Collaboration, Combination of $13 \mathrm{TeV} t \mathrm{ttH}$ analyses, https://twiki.cern.ch/twiki/bin/view/CMSPublic/TTHCombMoriond2016.

[7] CMS Collaboration, Search for top quark partners with charge $5 / 3$ at $\sqrt{s}=13 \mathrm{TeV}$, CMS-PAS-B2G-15-006.

[8] CMS Collaboration, Search for top quark-antiquark resonances in the all-hadronic final state at $\sqrt{s}=$ $13 \mathrm{TeV}$, CMS-PAS-B2G-15-003.

[9] CMS Collaboration, Run 2 SUSY Summary Plots, https://twiki.cern.ch/twiki/bin/view/CMSPublic/PhysicsResultsSus\#Moriond_2016.

[10] CMS Collaboration, Search for new physics in high mass diphoton events in $3.3 \mathrm{fb}^{-1}$ of proton-proton collisions at $\sqrt{s}=13 \mathrm{TeV}$ and combined interpretation of searches at $8 \mathrm{TeV}$ and $13 \mathrm{TeV}$, CMS-PAS-EXO-16-018.

[11] CMS Collaboration, Search for resonant production of high-mass photon pairs in proton-proton collisions at $\sqrt{s}=8$ and $13 \mathrm{TeV}$, PRL 117 (2016) 051802 .

[12] CMS Collaboration, Combination of searches for high-mass resonances in Z $\gamma$ final states in proton-proton collisions at $\sqrt{s}=8$ and $13 \mathrm{TeV}$, CMS-PAS-EXO-16-021.

[13] CMS Collaboration, Search for resonant production of high mass photon pairs using $12.9 \mathrm{fb}^{-1}$ of proton-proton collisions at $\sqrt{s}=13 \mathrm{TeV}$ and combined interpretation of searches at 8 and $13 \mathrm{TeV}$, CMS-PAS-EXO-16-027.

[14] CMS Collaboration, Measurement of the $B^{+}$hadron production cross section in pp collisions at 13 $\mathrm{TeV}$, CMS-PAS-BPH-15-004. 
[15] CMS Collaboration, Quarkonium production cross sections in pp collisions at $\sqrt{s}=13 \mathrm{TeV}$, CMS-PAS-BPH-15-005.

[16] CMS Collaboration, $D^{0}$ meson nuclear modification factor in PbPb collisions at $\sqrt{s_{N N}}=5.02 \mathrm{TeV}$, CMS-PAS-HIN-16-001.

[17] CMS Collaboration, Observation of particle-species dependence and collectivity in azimuthal correlations of pp collisions at the LHC, CMS-PAS-HIN-16-010.

[18] CMS Collaboration, Active Channels in 2016, https://twiki.cern.ch/twiki/bin/view/CMSPublic/ActiveChannelsSummary.

[19] CMS Collaboration, Level-1 Jets and Sums Trigger Performance, CMS-DP-2016-034.

[20] CMS Collaboration, Pre-LHCP 2016 L1 muon trigger performance, CMS-DP-2016-024.

[21] CMS Collaboration, Selected heavy flavor distributions from CMS with 2016 data at $13 \mathrm{TeV}$, CMS-DP-2016-016.

[22] CMS Collaboration, Muon performance in early 2016 collision data, CMS-DP-2016-027.

[23] CMS Collaboration, CMS ECAL first results with 2016 data, CMS-DP-2016-031.

[24] CMS Collaboration, Jet energy scale and resolution performances with $13 \mathrm{TeV}$ data, CMS-DP-2016-020.

[25] CMS Collaboration, ETmiss performance in events with Gamma, Z and dijets using $0.8 \mathrm{fb}^{-1}$ of 2016 Data, CMS-DP-2016-017.

[26] CMS Collaboration, Tau identification for Run-2 and its performance with 2015 data, CMS-DP-2016-019.

[27] CMS Collaboration, Identification of b quark jets at the CMS Experiment in the LHC Run 2, CMS-PAS-BTV-15-001. 